\title{
HERRAMIENTAS PARA EL ANÁLISIS Y MONITOREO EN LAS REDES SOCIALES
}

\section{FERRAMENTAS PARA A ANÁLISE E MONITORAMENTO NAS REDES SOCIAIS}

\author{
Juan Prieto-jjpg@buc.ucm.es \\ Doutorado em Ciência da Informação - Universidade de Madrid \\ Faculdade de Direito da Universidade Complutense de Madrid
}

\section{Resumen}

Introducción: Las redes y asociaciones son utilizadas por las personas desde los principios de la humanidad y su análisis emerge preocupaciones de distintos sectores de la sociedad. El Análisis de Redes Sociales estudia la relación que existe entre las personas, la estructura social, se compara la presencia online de una empresa con su debida competencia etc. Tratase de un área que está emergiendo como imprescindible en los procesos de decisión para su capacidad de analizar e intervenir en el comportamiento de la estrutucturas.

Objetivo: Evaluar tres herramientas de Análisis de Redes Sociales para monitorear las conversaciones acerca de la organización "IFLA" (Federación Internacional de Asociaciones de Bibliotecarios) para medir el sentimiento de las mismas, administrar los esfuerzos sociales y relacionar los flujos entre las entidades y los grupos.

Metodología: Ensayo

Resultados: La propiedad más importante de una herramienta para el Análisis de Redes Sociales es el echo de que son libres y virtuales. Los resultados muestran que el número de búsquedas realizadas sobre la IFLA, desde 2004, es decreciente en relación con el número total de búsquedas que han sido realizadas en Google. Por otra parte, es interesante destacar que el interés regional no coincide completamente con Google Trends y Google Insight.

Conclusiones: El estudio analizó las herramientas especializadas en el registro de la actividad en las medias sociales, que tienen un alto potencial para el ejercicio del control en la esfera de las relaciones públicas y de la investigación básica y aplicada.

\section{Palabras clave}

Análisis de Redes Sociales. Sistemas de Inteligencia. Relaciones Sociales. Internet. Redes de Investigación Científica. 


\section{INTRODUCCIÓN}

La creación de asociaciones es algo constitutivo del ser humano desde el inicio de su aparición y compartir conocimiento es una de los principales objetivos de las sociedades y comunidades (BARABASI, 2002).

La columna vertebral de la sociedad de la información, Internet, ofrece innegables cualidades de intercambios de conocimiento entre la sociedad. De hecho, las redes sociales se perfilan como el modelo de mayor crecimiento y más aun con la penetración de Internet móvil (GARCíA HERVÁS, 2010).

El conocimiento de la estructura del entorno es algo esencial para percatarse de donde te encuentras, con quien te relacionas, las tendencias, las predicciones, etc. Analizando las redes sociales se permite determinar lo que puede y no puede pasar, los comportamientos de ciertos colectivos, etc.

El análisis en redes sociales (de ahora en adelante ARS) es una actividad que está brotando como imprescindible en los procesos de toma de decisiones de instituciones, empresas, personales, de centros de investigación, etc.

El proceso interactivo de la Web 2.0 desarrolla redes sociales que se comunican a través de Internet donde las personas o instituciones aportan y esperan recibir información útil de características o similitudes afines.

Mediante colaboraciones involuntarias entre agentes (debido a fenómenos de estigmergia) o voluntarias, se detecta conocimiento e inteligencia, el cual se mostrará mediante diferentes herramientas comentadas en la investigación.

\section{REDES SOCIALES}

La estructura de las redes ha evolucionado a lo largo de la historia, los humanos son sociales y las organizaciones adquieren nuevos paradigmas y tipológicas.

Se puede definir una red social como una organización o estructura generada a través de las relaciones de diferentes actores (personas, instituciones, organizaciones, sociedades, etc.), todas ellas deben poseer o estar vinculadas a ciertas particularidades 0 rasgos comunes con el fin de poder relacionarse entre sí.

En la actualidad destacan las redes creadas a través de las nuevas tecnologías, de Internet, de interacción social y que son en las que se centra la investigación. 
Ferramentas para a ánalise e monitoriamento nas redes sociais

La particularidad más notable de las mismas es la conexión online para acceder a ellas y el constante dinamismo que se genera. Las comunidades se representan mediante las conexiones personales que los usuarios disponen los unos de los otros (ROSMARTÍN, 2009).

La primera red social, classmates.com, surgió en 1995 y fue ideada por Randy Conrads a partir de ese momento emergen numerosas redes, principalmente para relacionar o conectar a amigos, compañeros o colegas.

Con la generación de la Web 2.0, aproximadamente en el año 2004, se produce una explosión en el campo de las redes sociales donde las temáticas y contenidos abarcan todas las áreas y donde se generan nuevas formas de constituir y conservar relaciones sociales.

Es importante destacar la gran cantidad de medios sociales ofrecidos en la red. Por ello, las clasificaciones se hacen necesarias con el fin de adecuarse para el desarrollo de análisis exhaustivos. Cabe destacar las redes sociales horizontales donde tiene cabida cualquier temática bien de ocio, laboral, deportes, etc. (Facebook, Twitter, Friendster, MySpace, YouTube, etc.) y las verticales que se especializan en temas concretos (Check My Ride, Xing, BabyCenter, etc), estas últimas, crecen a mayores porcentajes que los primeros (FUMERO; GARCÍA, 2008, p. 34).

\section{MEDICIÓN EN LOS MEDIOS SOCIALES}

Con el desarrollo anterior se dice que el análisis de redes sociales es la cartografía y la medición de las relaciones y los flujos entre las personas, grupos, organizaciones o equipos (VALDIS, 2002) siendo necesarias en los procesos de toma de decisiones (WASSERMAN, 1994).

Una vez evaluada o transformada la información y procesado el conocimiento extraído a partir del uso de determinadas herramientas, el objetivo será obtener el mayor nivel de inteligencia personal, en los centros de investigación o en empresas tecnológicas, con el fin de asesorar y dirigir políticas hacia las marcas privadas, personales y las Administraciones Públicas.

Las herramientas tecnológicas han fortalecido este desarrollo mediante la fusión de las ideas y opiniones ofrecidas en las redes sociales, aprovechando su gran para obtener datos y realizar tareas de vigilancia. No cabe duda que para conocer lo que está pasando 
en el tejido social se necesita contar con instrumentos avanzados de tracking para realizar diagnósticos y monitorización de acciones en las redes sociales. La aproximación desde una metodología observacional es fundamental, puesto que se recoge lo que ocurre en el medio social interactivo.

Existen una gran variedad de proveedores de investigación que ofrecen herramientas que reflejan el entorno social y en común tienen el análisis de los contenidos generados por los usuarios o consumidores CGM (Consumer-Generated Media).

Por otro lado, en la red se dispone de un gran número de herramientas gratuitas que realizan el seguimiento de los contenidos sociales en todos los campos de la red.

Por limitaciones de espacio, se analizarán varias herramientas de monitorización, con el fin de ajustar las cualidades de cada una de ellas a las demandas del usuario. Unas herramientas pueden monitorizar conversaciones a lo largo y ancho de la red sobre un tema específico, marca o persona y otras, centrarse en el análisis de determinadas aplicaciones de una forma simultánea. Ante esta situación se analizarán tres herramientas, la primera de ellas, Socialmention busca en variados espacios. La segunda opción reúne dos herramientas de Google, Trends e Insights y por último, tras la cada vez mayor demanda de Twitter, Follow the Hashtag.

Para facilitar la labor de análisis, se ha decidido realizar búsquedas de la palabra clave "IFLA" debido a la popularidad de la misma en el área y a la amplitud del marco temporal a analizar.

\section{HERRAMIENTAS PARA EL ANÁLISIS DE INTELIGENCIA EN REDES SOCIALES}

Socialmention: Es un motor de búsqueda totalmente gratuito con contenido generado por el usuario en Blogs, Microblogs, foros, imágenes, videos, noticias, comentarios, eventos y la posibilidad de realizar la búsqueda en todos a la vez.

Con fecha 1 de septiembre se ha testado el posicionamiento en la red de la palabra clave "IFLA" (International Federation of Library Associations).

Esta herramienta además cuantificar el número de entradas y el acceso a los comentarios realiza un análisis semántico de los contenidos, de modo que permite realizar una valoración más descriptiva del sentido de las menciones, destacando: 
- Strength (fuerza): 2\%. Es la probabilidad con la que se está debatiendo en los medios de comunicación social sobre la IFLA. Para calcular el porcentaje se emplea un cálculo simple: frase mencionada en las últimas 24 horas, dividida por el total de posibles menciones.

- Sentiment (sentimiento): 13:1. El sentimiento es la proporción de menciones positivas sobre las que son negativas. Por lo que de cada diez menciones una es negativa.

- Passion (pasión): 37\%. La pasión es una medida de la probabilidad de que los individuos que hablan de su marca, lo harán varias veces. Las menciones positivas sobre la IFLA, han sido realizadas por diferentes personas. El porcentaje se calcula mediante las palabras clave más utilizadas y el número de veces mencionadas, en definitiva es el número de menciones por sentimiento.

- Reach (alcance): 26\%. Es una medida del radio de influencia. Es el número de autores únicos que referencian a la IFLA dividido por el número total de menciones.

Se observa que el motor de búsqueda de socialmention es muy completo trabajando con más de una centena de redes sociales, entre las cuales se encuentran Twitter, Facebook, Linkedin,YouTube, StumbleUpon, Digg o Google, permitiendo la exportación a hojas de cálculo de la información obtenida.

Como dato positivo cabe destacar la eliminación de búsqueda en los scraper sites.

Asimismo el resultado de búsqueda indica las Top Keywords relacionadas con la cadena buscada, los Top users que las generaron, el Top Hashtag (siendo wlic2011, que obviamente coincide con la reunión anual de la IFLA, celebrada en el año 2011 en Puerto Rico) y las fuentes relacionadas, que encabezan por Twitter.

Por último, es posible suscribirte vía RSS a una búsqueda concreta. Interesante para no perder de vista qué se dice en Internet sobre un determinado concepto.

Destacar, que en pocos minutos es posible realizar un seguimiento y medir fácilmente lo que la gente está diciendo de uno mismo, de otro, de una empresa, un producto o cualquier otro tema a través de los medios de comunicación social de la Web.

Gráficamente obtenemos: 
Figura 1 - Gráficos de las búsquedas del termino “IFLA" el día 1/09/11.

\begin{tabular}{|l|r|}
\hline $\begin{array}{l}\text { 2\% } \% \\
\text { strength }\end{array}$ & $\begin{array}{c}13: 1 \\
\text { sentiment }\end{array}$ \\
\hline $\begin{array}{c}37 \% \\
\text { passion }\end{array}$ & $\begin{array}{r}26 \% \\
\text { reach }\end{array}$ \\
\hline 1 hours avg. per mention \\
\hline last mention 2 hours ago \\
\hline 155 unique authors \\
\hline 25 retweets \\
\hline $\begin{array}{l}\text { Sentiment } \\
\text { positive } \\
\text { neutral } \\
\text { negative }\end{array}$ \\
\hline
\end{tabular}

Top Keywords
lang
yung
kita

\section{Top Users}

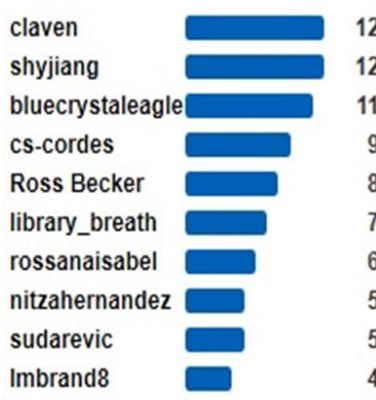

Fuente: Socialmention.

Top Hashtags
wlic2011
ebooks
libraries
ifla
digitalhumanitie
rfid
austl
ubclibrary
wlic2013
copyright

\section{Sources}

$\begin{array}{lll}\text { twitter } & 86 \\ \text { delicious } & 48 \\ \text { photobucket } & \\ \text { facebook } & & 39 \\ \text { picasaweb } & & 25 \\ \text { plurk } & & 25 \\ \text { google_blog } & 16 \\ \text { google_video } & & 10 \\ \text { Yahoo News } & 10 \\ \text { bing } & 6 \\ \text { metacafe } & \text { I } & 2 \\ \text { reddit } & & 2 \\ & & 1\end{array}$

En segundo lugar, se realiza una interesante exploración de dos herramientas de Google, debido a que es el buscador más utilizado en Internet sus resultados suelen mostrar autenticidad, Google Insighits y Google Trends.

Con fecha 1 de septiembre se ha testado el posicionamiento en la red de la palabra clave "IFLA".

Google Trends: proporciona una relevante información sobre la relevancia que tienen los términos de búsqueda en la red sin indicar concretamente el contenido social, permitiendo visualizar gráficamente la evolución de búsquedas en un determinado periodo de tiempo (desde el año 2004 hasta la actualidad) pudiendo analizar comportamientos sociales o posibles estacionalidades entre otra información.

En la parte superior del gráfico aparecen las variables de búsqueda mostradas a lo largo del tiempo (eje abscisas) y en el gráfico inferior las noticias publicadas sobre la IFLA. En el eje vertical representa la frecuencia con la que se ha buscado el término globalmente. 
Acotando la muestra a ciclos anuales es posible percibir el despunte de la estacionalidad de los periodos en que se celebran los congresos anuales, generalmente en agosto. Otra de las características de la herramienta es la posibilidad de comparar hasta cinco términos simultáneamente y acceder al acotamiento geográfico regional, de ciudades y del lenguaje empleado a través de gráficos.

Figura 2 - Gráfico de la evolución del termino "IFLA" desde el año 2004.

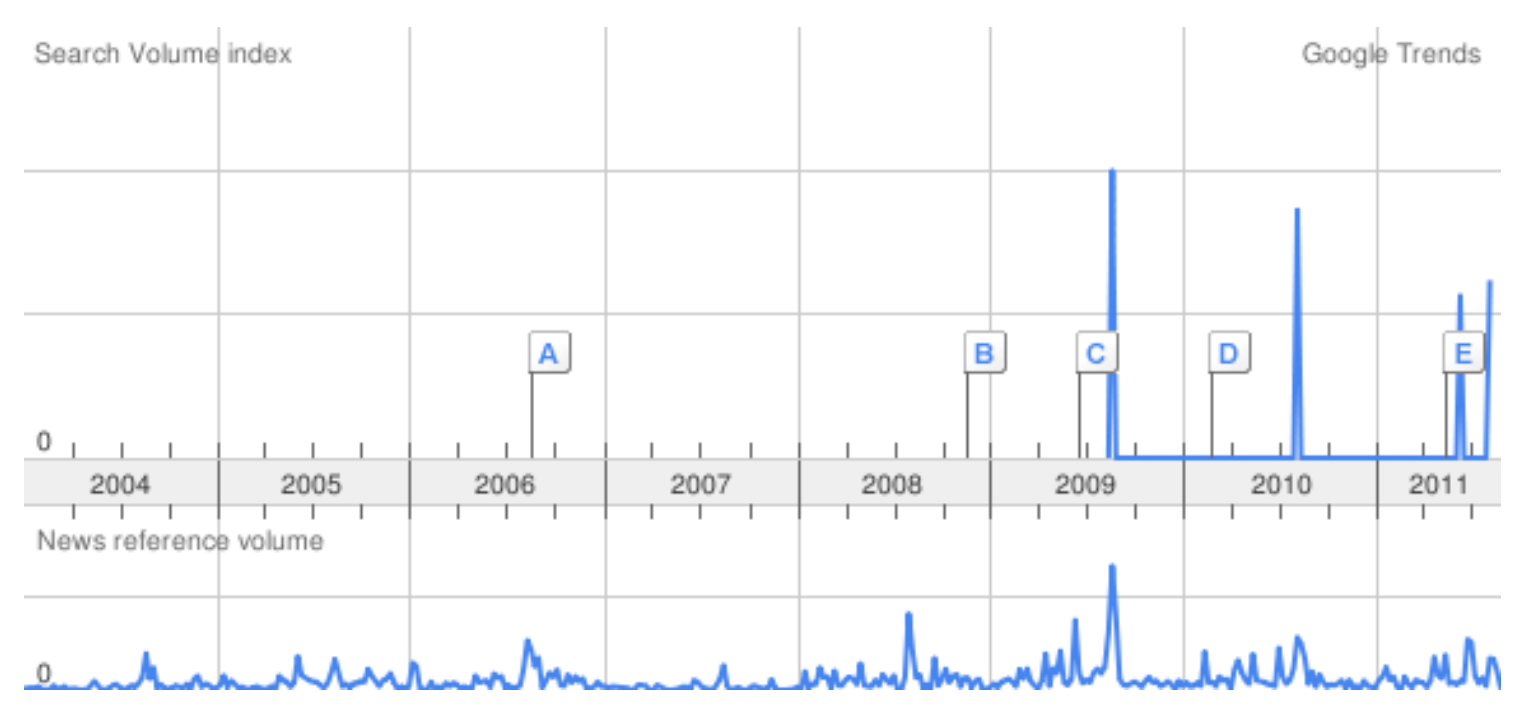

Fuente: Google Trends.

Google Insights: posee una gran similitud en cuanto a la idea y a la funcionalidad que Google Trends, pero ofrece más datos sobre las palabras clave y precisa la geolocalización de los rastreos.

Cabe la posibilidad de cotejar patrones de volumen de búsqueda en determinadas áreas geográficas, intervalos de tiempo, categorías y propiedades analizando que zona está más interesada en el término examinado. Gráficamente se accede al resultado acotado temporalmente desde el año 2004 hasta la actualidad y representando en el eje vertical la frecuencia con la que se ha buscado el término. En la tabla se muestra un mapamundi con el índice de volumen de búsquedas representado por diferentes colores. 
Juan Prieto

Ferramentas para a ánalise e monitoriamento nas redes sociais

Interés de búsqueda en la Web: IFLA

Figura 3 - Evolución de las búsquedas del termino "IFLA" desde el año 2004.

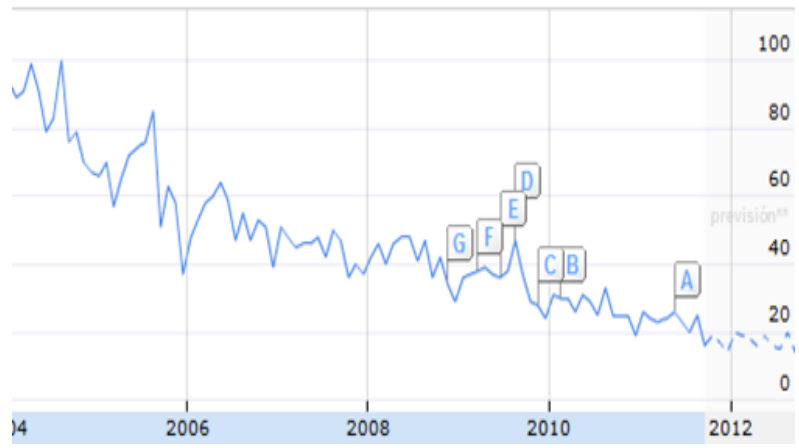

Fuente: Google Insights.

http://www.google.com/insights/search/
Interés regional:

Figura 4 - Distribución por países del volumen de las búsquedas del termino "IFLA".

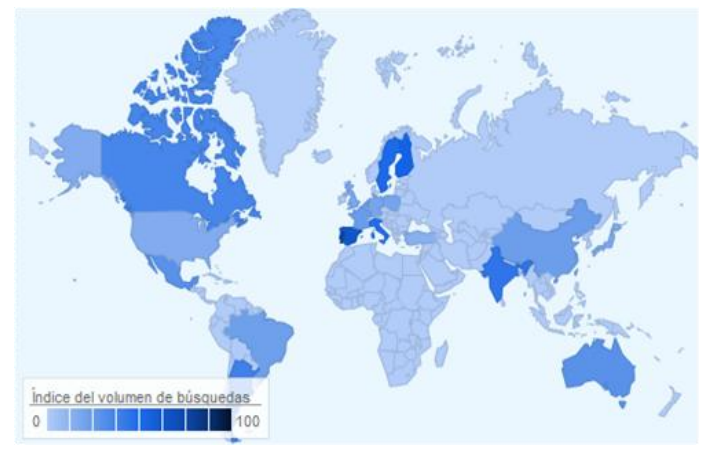

Fuente: Google Insights.

cat $=0-11 \& \mathrm{q}=\mathrm{IFLA} \& \mathrm{cmpt}=\mathrm{q} \# \mathrm{cat}=0$ -

\section{$11 \& \mathrm{q}=\mathrm{IFLA} \& \mathrm{cmpt}=\mathrm{q}$}

Los resultados demuestran que el número de búsquedas que se han realizado, sobre la IFLA, desde el 2004 es decreciente en relación con el número total de búsquedas que se han hecho en Google. Por otro lado es curioso destacar que el interés regional no coincide plenamente entre Google Trends y Google Insight.

Como observación comentar que las interfaces de Google son muy sencillas, manejables y fiables al pertenecer al laboratorio Google. Aportando información muy provechosa complementando a otras.

Existen infinidad de herramientas que trabajan sobre Twitter, bien para administrar la cuenta, para gestionar a tus seguidores, para saber de que se habla, el seguimiento de hashtags, para calcular la influencia, gestionar eventos, etc.: como por ejemplo klout, Twendz, Twitter Sentiment, TwitterCounter, etc. Habiéndose elegido Follow the Hashtag creada por DNOiSE la cual permite conocer, conversaciones en Twitter mediante gráficas útiles y coherentes.

Como en las anteriores herramientas se ha introducido el hashtag IFLA, (\#IFLA) durante la semana del 26 de agosto al 1 de septiembre de 2011 y como resultado se dispone de 169 tweets, con una frecuencia de 1.13 tweets por hora de 138 twitteros diferentes, siendo el más activo @ABESelsalvador con 6 tweets y, mediante el diagrama de puntos o de burbujas generado, es posible apreciar la jerarquía de importancia de los tweets. 
La precisión y limitación de la herramienta depende actualmente de la API de búsqueda de Twitter. Una de las restricciones que impone es que "como mucho" se remontará a los últimos 1500 tweets o 30 días pasados por cada keyword.

En la zona de búsqueda y control permite introducir los parámetros deseados y contrastar hasta 4 keywords o hashtag al mismo tiempo, muy útil para realizar benchmarking. También es posible controlar la cantidad de búsquedas hechas y representadas, el lenguaje y la localización y mediante la función random es posible acceder a diferentes gráficos.

Figura 5 - Diagrama de burbujas generado por la búsqueda del termino "IFLA" del 26/08/11 al 01/09/11.

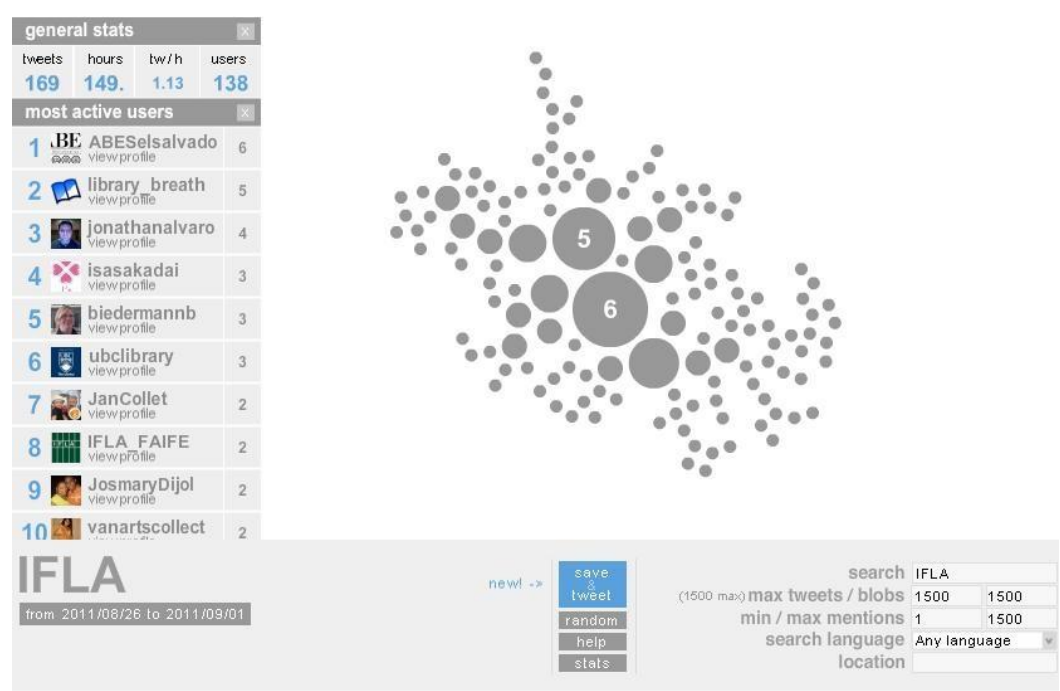

Fuente: Follow the Hashtag.

\section{CONSIDERAÇÕES FINAIS}

La propiedad más rica de una herramienta que analice redes sociales es el hecho de que éstas son libres y virtuales, permitiendo que funcionen en los ámbitos más adversos con problemáticas de un sector geográfico o ideológico, conjugando el sistema de comunicación con la motivación social.

Los usuarios son dueños de los contenidos de las redes, por lo que el ARS se da cuenta y se apodera de ellos. Las redes sociales son más que un punto de encuentro, siendo necesario el empleo de diferentes aplicaciones virtuales capaces de coordinar y manifestar el comportamiento de las personas y las marcas transformándose los resultados en un componente inteligente. 
En el estudio se examinan cuatro herramientas especializadas en el registro de la actividad en medios sociales, las cuales tienen un alto potencial para el ejercicio del seguimiento en el ámbito de las relaciones públicas y de la investigación tanto básica como aplicada. Hay un beneficio añadido de las herramientas que incluyen un analizador semántico que permite analizar los contenidos para su clasificación creando indicadores de la actividad. Estos indicadores resultan especialmente adecuados para llevar a cabo comparativas temporales o de competencia.

La posibilidad de graficar las estructuras sociales y medir sus propiedades a partir de sofisticados programas de software, de muchas de estas herramientas, permiten de una manera comprensible y sucinta acceder a los resultados de la comunicación, colaboración, transacción, valoración, o cualquier otro tipo de relación que se desarrolle a través de medios presenciales o virtuales.

Se ha observado una gran actividad y participación en las redes destacando algunos líderes de opinión y es ahí donde el ARS selecciona y filtra la información con el fin de distinguir el conocimiento.

\section{REFERÊNCIAS}

BARABASI, A. L. Linked: the new science of networks. Cambridge, MA: Perseus, 2002.

FUMERO, Antonio; GARCIA, Miguel. Redes sociales: contextualización de un fenómeno "dos-punto-cero". Telos: Cuadernos de Comunicación e Innovación, n. 76, p.56-68, 2008.

GARCIA HERVAS, Jesús Galván. Redes sociales en el móvil.Telos: Cuadernos de Comunicación elinnovación, n. 83. p. 8-12, 2010.

ROS-MARTÍN, Marcos. Evolución de los servicios de redes sociales en Internet. El Profesional de La Información, v. 18, n. 5, p.552-558, sep./oct. 2009.

VALDIS, E. Krebs. Mapping Networks of Terrorist Cells. Connections, v. 24, n.3, p. 4352, 2002.Disponível em: <http://www.sfu.ca/ insna/Connections-Web/Volume243/Valdis.Krebs.web.pdf. Acesso em: 12 maio 2012.

WASSERMAN, S.; FAUST, K. Social network analysis: methods and applications. Cambridge :Cambridge University Press, 1994. 
Title

Tools for Social Networks monitoring and analysis

\section{Abstract}

Introduction: Networks and associations have been used by human beings since the dawn of mankind, and their analysis raises concerns in several sectors of society. Social Network Analysis studies the relationships among people and the social structure, it compares e-commerce with company competence, and so on. It is an emerging area that is essential to decision-making processes due to its ability to analyze and interpose in the behavior of the structures.

Purpose: To analyze three Social Network Analysis tools for monitoring conversations about the IFLA (International Federation of Library Associations) in order to measure their sentiment, to manage social efforts and to relate the flows between entities and groups.

Methodology: Essay.

Results: The most important property of the Social Network Analysis tools is the fact that they are free and virtual. On the one hand, results show that the number of searches dated from 2004 about the IFLA is decreasing in relation to the total number of searches that have been made in Google. On the other hand, it is interesting to highlight that the regional interest does not fully coincide between Google Trends e Google Insight.

Conclusions: The study analyzed tools specialized in recording the activity on social media, which have a high potential for conducting monitoring in the field of public relations as well as in a basic and applied research context.

\section{Key words}

Social Networks Analysis. Intelligence systems. Social Relations. Internet. Scientific Research Networks.

\section{Título}

Ferramentas para a análise e monitoramento nas redes sociais

\section{Resumo}

Introdução: As redes e associações são usadas pelos seres humanos desde os primórdios da humanidade e sua análise levanta preocupações de diversos setores da sociedade. A Análise de Redes Sociais estuda a relação que existe entre uns e outros, a estrutura social, compara a presença online de um negócio com a competência, etc. É uma área que está surgindo, essencial nos processos de tomada de decisão por sua capacidade de analisar e intervir nos comportamentos das estruturas.

Objetivo: Avaliar três ferramentas de Análise de Redes Sociais para monitorar as conversas sobre a Organização "IFLA" (International Federation of Library Associations) para medir o sentimento das mesmas, gerenciar os esforços sociais e relacionar os fluxos entre as entidades e grupos.

Metodologia: Ensaio.

Resultados: A propriedade mais importante de uma ferramenta para analisar as redes sociais é o fato de serem livres e virtuais. Os resultados demonstram que o número de buscas realizado sobre a IFLA, desde 2004 é decrescente em relação com o número total de buscas que têm sido 
Juan Prieto

Ferramentas para a ánalise e monitoriamento nas redes sociais

feitas no Google. Por outro lado é interessante destacar que o interesse regional não coincide plenamente entre Google Trends e Google Insight.

Conclusões: No estudo foram analisadas ferramentas especializadas no registro da atividade em mídias sociais, que têm um elevado potencial para o exercício de acompanhamento no âmbito das relações públicas e de pesquisa básica e aplicada.

Palavras-chave: Análise de Redes Sociais. Sistemas de Inteligência. Relações Sociais. Internet. Redes de Pesquisa Científica.

Recebido em: 07/09/2011

Aceito em: 21/07/2012 